\title{
Exit-Surface Dependency of Defocus measured by Off-axis Electron Holography
}

\author{
Michael Lehmann \\ University of Dresden, Triebenberg Laboratory, D-01062 Dresden, Germany \\ In high-resolution electron microscopy (HRTEM), the object under investigation is illuminated by a \\ plane electron wave. According to the dynamical interaction with the object, the object exit-wave is \\ modulated in amplitude and phase. This object exit-wave is imaged by the objective lens and, due to \\ its wave aberration, amplitude and phase information is mixed up resulting in the so-called image \\ wave. On the detector, only the intensity, i.e. the amplitude squared, is recorded while the phase \\ information is lost.
}

At atomic dimensions, the image contrast strongly depends on defocus. Therefore, without exact knowledge about defocus (and all other aberrations), the interpretation of HRTEM-images may be misleading. In addition, there is another point, which has to be taken into account: Normally, it is assumed that the surfaces of the sample are parallel to the electron wave. However, depending on preparation, experience, and material, the surfaces of real-world samples are often poorly defined. Especially for complex unit cells with many different atoms, thickness variations of the crystal cause reasonable strong position-dependent contrasts. An even more severe effect, however, have deviations of the exit-surface from the parallel electron wave since they additionally cause a position-dependent defocus. In a conventional intensity image, both contributions can hardly be distinguished.

Holographic methods like off-axis electron holography allow correcting the coherent wave aberration of the objective lens. Moreover, the electron wave can freely be back-propagated to any plane. Therefore, deviations of the exit-surface from the parallel electron wave can be numerically counterbalanced by means of defocus resulting in the locally true object exit-wave. The drawback is the defocusing of other parts of the field of view, since the application of the correcting phase plate in Fourier space can only be performed for isoplanatic aberrations.

Fig. 1 (left) shows the from an off-axis electron hologram reconstructed object exit-wave of a [110]oriented GaAs wedge-shaped crystal. The wedge angle is 45 degree and the exit-surface is parallel to the electron wave. The typical dumbbell-contrast can be observed in the thin as well as in the thicker areas of the crystal. Taking the same sample, but inserting it upside down into the specimen holder, yields a strongly inclined exit-surface hence defocus-dependency (Fig. 1 right). Now, the dumbbell contrast can either be observed at the thin edge or, after underfocusing by $14 \mathrm{~nm}$ according to the geometry of the sample, in thicker region beyond the first extinction thickness. Even in a perfectly corrected electron microscope without coherent aberrations, this geometry effect of the exit-surface would hamper the image interpretation.

\section{Acknowledgements:}

Many thanks are due Prof. Henny W. Zandbergen and Ides Büscher from the TU Delft for providing the wedge-shaped GaAs sample. The discussions at the Triebenberg Lab, especially with Prof. Hannes Lichte and Dr. Dorin Geiger, and the financial support by the German Research Foundation are gratefully acknowledged. 


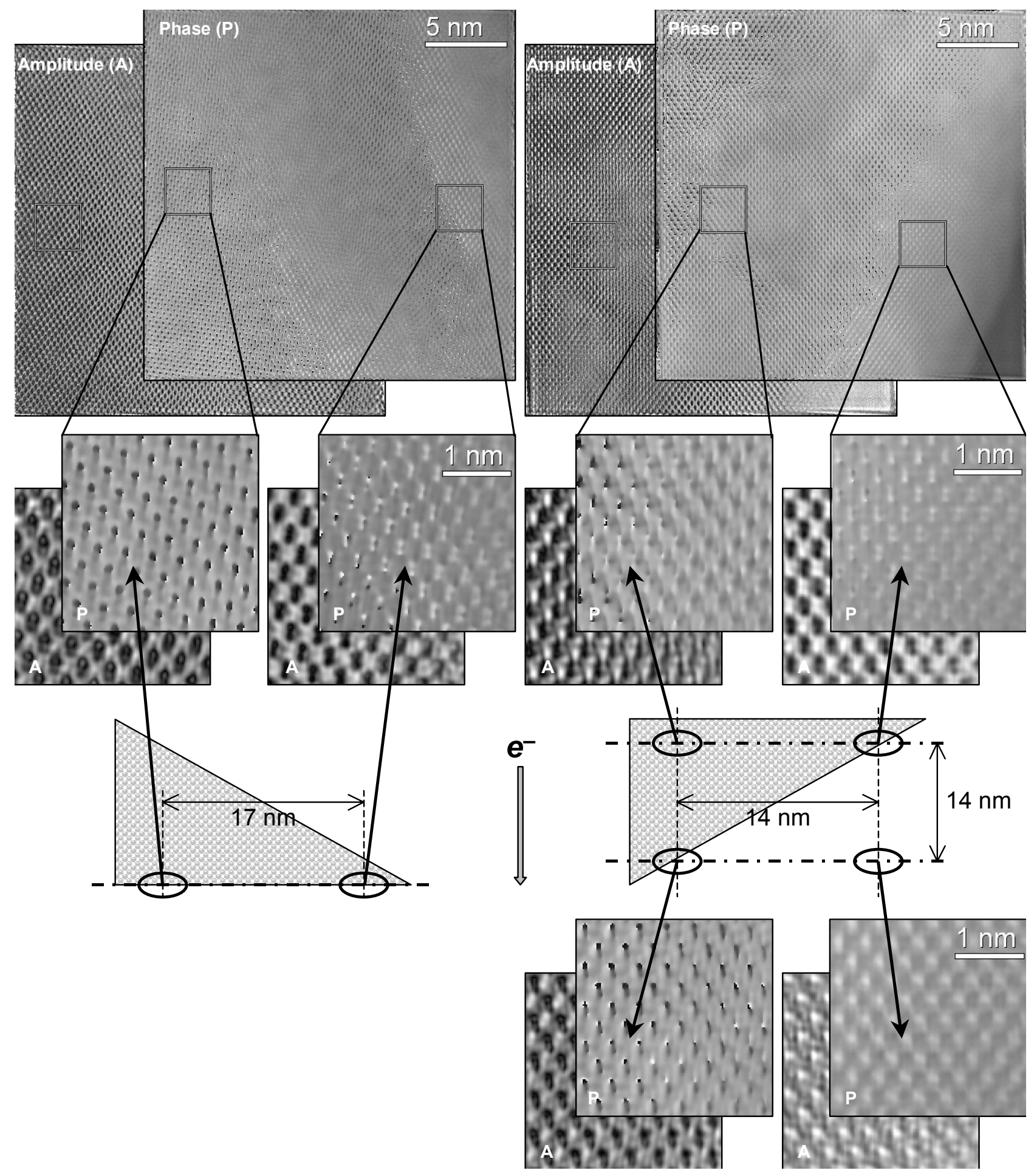

Fig. 1: [110]-oriented GaAs wedge-shaped crystal with 45 degree wedge angle. All images are slightly noise-reduced by Fourier-filtering. Left: Exit-surface is parallel to incident plane electron wave hence contrast differences in amplitude (A) and phase (P) only due to thickness differences. Right: Exit-surface is strongly inclined hence contrasts also depend on the height of the reconstructed exit-plane. According to the geometry of the sample, defocusing by $14 \mathrm{~nm}$ shifts the region of dumbbell-contrast between thin (top) and thick (bottom) sample areas. 